\title{
Wer nur den Preis sieht und den Wert nicht erkennt, wird sich mit der Umsetzung schwertun
}

\section{Christoph Bosshard}

Präsident VSAO

Verband Schweizerischer Assistenz- und Oberärztinnen und -ärzte

Korrespondenz:

Dr. med. Christoph Bosshard VSAO

Bahnhofplatz $10 \mathrm{~A}$

Postfach 8650

CH-3001 Bern

bosshard@vsao.ch
... und nun noch dies! Stetige Verknappung der Finanzen, immer höhere Anforderungen an Fallzahlen, immer mehr Administration, und dies bei $75 \%$ Personalkosten des Gesamtaufwandes. Wie sollen wir Ärzte dieses Dilemma nur lösen? Daran gewohnt, alle Arten von Problemen selbst an die Hand zu nehmen, packen wir nun auch dieses an und versuchen, unsere alten Klinikabläufe nach den neuen Vorgaben zu organisieren. Das Folgeproblem ist rasch ersichtlich: Die Stellenpläne explodieren. Falls überhaupt finanzierbar - wo finden wir denn all die Assistenz- und Oberärztinnen und -ärzte, wenn wir jetzt schon nur mit bis zu $40 \%$ ausländischer Unterstützung funktionieren? Mittels Aufstockung der Stellen kann die Umsetzung des Arbeitsgesetzes also nicht funktionieren. Es macht auch wenig Sinn, dass einfach immer mehr Ärzte die unverändert ineffizienten (da zu wenig hinterfragten) Strukturen mittragen.

Es ist somit höchste Zeit für eine tiefgreifende Analyse nicht nur der Klinik, sondern des gesamten Spitals, ja der Zusammenarbeit im Gesundheitswesen über die Spitalmauern hinaus. Der VSAO hat dazu Ansätze entwickelt und definiert, wo Optimierungspotential besteht (siehe Kästchen). Von Anfang an muss jedoch klar sein: Ohne Aufwand und etwas Mut lässt sich keine Veränderung erreichen. Auch sind die Ärzte nicht alleine verantwortlich, nein, alle schnittstellenbildenden Bereiche sind füreinander verantwortlich, zuoberst die Spitalgeschäftsleitung.

Dank der gegenseitigen Verantwortung werden Prozesse in Gang gesetzt, die von ebenso gegenseitiger Wertschätzung geprägt sind, weil

\section{Schwerpunkt Arbeitsinhalte}

- Gute Einführung bei Beginn einer neuen Stelle (auch in Administrativem, z. B. Computer, wie kurz und prägnant Austrittsberichte sein sollen, wann Kurzberichte erlaubt sind usw.)

- Arbeiten an sich: Ist es notwendig, dass ein Arzt/eine Ärztin diese Arbeit erledigt? Entsprechen die Arbeitsinhalte der Qualifizierung der Mitarbeiter/innen?

- Schaffung/Optimierung von Stations-/Ärztesekretariaten, im Sinne eines Case-Managements mit sinnvoller Aufgabenbündelung und Kompetenzen es eben nicht egal ist, was die andern Partner in einem Prozessablauf tun. Im weiteren ist die ärztliche Arbeitskapazität nicht nur zu teuer für nichtärztliche oder ineffizient organisierte Aufgaben, sie ist in Zukunft auch immer weniger verfügbar. Das OBSAN ortet im Jahr 2030 einen Mangel von 30\% Ärzten im Bereich Grundversorgung und 15-30\% im Bereich Spezialisten. Diese Tendenz wird vor den Spitälern nicht haltmachen. Wer meint, es werde alles so weitergehen wie bisher, steckt den Kopf in den Sand.

Von dieser Warte betrachtet erhält das Arbeitsgesetz im Gesundheitswesen einen positiven Impetus und kostet nicht nur Geld und Nerven. Dank der Vorschriften werden längst überfällige Entwicklungen in die Wege geleitet mit dem Ziel einer nachhaltigen, tiefgreifenden und breitenwirksamen Optimierung.

Die Arbeitszeitbestimmungen gründen auf arbeitsmedizinischen Studien und führen somit auch zu mehr Sicherheit und Qualität im klinischen Alltag, da Fehler und Patientenaversionsreaktionen wegen Übermüdung reduziert werden. Auch haben Ärztinnen und Ärzte Anrecht auf ein soziales Umfeld, welches ihnen nicht zuletzt zur Sozialkompetenz verhilft, welche sie in ihrem klinischen Alltag ihren Patienten und Mitarbeitern gegenüber dringend benötigen.

Nicht zuletzt ermöglicht die 50-StundenWoche den Assistenz- und Oberärzten eine adäquate Weiterbildung, da die dazu notwendigen Freiräume geschaffen werden, und Weiterbildner wie auch Weiterzubildende nicht in uferloser Dienstleistung ihre Kräfte aufreiben.

Aufgrund der genannten Faktoren wie Optimierung der Prozesse unter Einbezug der verbesserten Zusammenarbeit, Steigerung der Sicherheit und Qualität und Erhöhung der Arbeitszufriedenheit würde es mich nicht erstaunen, wenn uns das Ganze am Ende mehr bringt als kostet, d. h. selbst aus ökonomischer Sicht von Vorteil ist.

Das Arbeitsgesetz bringt uns schliesslich etwas, unter dessen Mangel wir stets leiden, nämlich Zeit. Es lehrt uns den bewussten Umgang mit dieser Dimension. Ich wünsche uns allen Zeit nicht einfach zum Vertreiben! 


\section{Schwerpunkt Arbeitsorganisation}

- Optimale Einsatzplanung, sowohl kurz- wie mittelfristig, z. B. mittels geeigneten Informatiktools (PEP)

- Piepserfreie Stunden (Piepsertriage z. B. durch Sekretariat), reservierte «Bürostunden» einführen $\rightarrow$ deutlich höhere Produktivität!

- Arbeitsabläufe anschauen - die Prozesse/Schnittstellen ständig optimieren, den individuellen Mitarbeiter(inne)n anpassen

- Kernzeiten definieren mit Anwesenheit aller, flexible Randzeiten, um Arbeitszeit wechselndem Arbeitsanfall anpassen zu können

- Rapport-/Weiterbildungszeiten überdenken, Teilnehmende überdenken, evtl. Rapporte weglassen?

- Schemata für Routineuntersuchungen entwickeln inkl. Vorlagen/Textbausteinen fürs Berichtswesen, vermehrter Einsatz von Kurzberichten mit den wesentlichen Informationen

- Diktiermöglichkeit in unmittelbarer Nähe der Sprechstunden einrichten

- Wo noch nicht eingeführt: Nachtarztsystem, möglichst über Klinikgrenzen hinweg

- Mittagspause: Sind diejenigen definiert, welche abrufbar sein müssen? Besteht für die anderen die Möglichkeit, die Mittagspause ungestört zu verbringen oder das Haus zu verlassen?

\section{Unbegründeter Widerstand gegen das Arbeitsgesetz}

\section{Rosmarie Glauser}

Fürsprecherin, Politische Sekretärin VSAO
Korrespondenz: Rosmarie Glauser VSAO

Bahnhofplatz 10 A Postfach 8650 CH-3001 Bern

glauser@vsao.ch
Seit dem ersten Januar 2005 unterstehen die Assistenzärztinnen und -ärzte den Schutzbestimmungen des Arbeitsgesetzes (ArG). Parallel dazu drang ins Bewusstsein, dass das ArG für viele Oberärztinnen und -ärzte und andere Spitalangestellte seit langem gegolten hätte oder dass es infolge neuer Spitalstrukturen neu gelten wird.

Mehr als drei Jahre später polarisiert das Gesetz immer noch, und nach wie vor gibt es massive Verstösse gegen die gesetzlichen Bestimmungen. Mögliche Gründe dafür sind:

- Die Spitäler stehen unter enormem Kostendruck, der an die Leitenden Ärzte weitergegeben wird.

- Veränderung ist unbequem und braucht in einem ersten Schritt Ressourcen.

- Im ärztlichen Bereich hält sich teilweise immer noch der Anwesenheitskult. Lange Arbeitszeiten gelten bei vielen Chefärzten als Ausdruck von Leistungsfähigkeit, Leistungswille und ärztlichem Ethos.

- Assistenzärztinnen und -ärzte sind auf ihrem Weg zum Facharzttitel in besonderer Weise von ihren Vorgesetzten abhängig und daher gezwungen, deren Bedingungen zu akzeptieren.

- Es wird den jungen Ärztinnen und Ärzten schwergemacht, die Schutzbestimmungen des Arbeitsgesetzes als gute Sache - auch für die Sicherheit der Patienten - zu sehen, und sich für ihre Rechte einzusetzen. Hier eine Auswahl der Standardvorhaltungen und unsere Antwort darauf:

Du bist zu langsam, andere schaffen das in der gesetzlich vorgegebenen Arbeitszeit Sollte das wirklich der Fall sein, sind die Führungsqualitäten des Vorgesetzten gefragt. Er muss prüfen, warum das so ist und welche Hilfeleis-

\section{Schwerpunkt Betriebskultur} und Mitarbeiterförderung

- Verbesserung der Motivation durch überprüfbare Zielvereinbarungen und Erarbeiten einer individuellen Laufbahnplanung

- Unterstützung im Zeitmanagement, laufende Förderung und Betreuung der Ärztinnen/Ärzte, Vermitteln von Anerkennung und ehrlichem, persönlichem Feedback

- Allgemeine Massnahmen zur Verbesserung der Mitwirkungsmöglichkeiten, Ideenmanagement (früher Vorschlagswesen) innerhalb jeder Klinik

- Teambildende Massnahmen: gesellige Anlässe, gemeinsamer Pausenraum 
tungen er geben kann und muss. Oft wird aber das ArG bei allen nicht eingehalten.

\section{Mit dem Arbeitsgesetz ist die WB nicht mehr gewährleistet}

Alle bisherigen Untersuchungen und Befragungen zeigen das Gegenteil. Die WB wird sogar als besser eingestuft. Lernen ist ein aktiver Vorgang, der um so besser funktioniert, je höher die Konzentration ist. Es ist nicht so, dass Erfahrung allein eine gute WB definiert. Die praktische Erfahrung muss mit Theorie vernetzt, aktiv aufgearbeitet und mit bestehendem Wissen verknüpft werden, um bleibende Spuren im Gedächtnis zu hinterlassen. Für diesen Aspekt verbessert das Arbeitsgesetz die Rahmenbedingungen, es öffnet Räume für Erholung und aktive Aufarbeitung.

Im chirurgischen Bereich ist die Übung von Eingriffen wichtig, aber auch das ist bei guter

\section{Schwerpunkt Unternehmensführung}

- Strategische Überprüfung der Dienstleistung, heisst: Definition von kurzund längerfristigen Zielen, die nachvollziehbar und handlungsleitend sind und offen kommuniziert werden. Sich folgende Fragen stellen:

- Wofür besteht ein Leistungsauftrag? Welches ist für die gute Behandlungsqualität zwingend notwendig?

- Was können andere besser, was kann dem/der betreuenden Hausarzt/ -ärztin zurückgegeben werden?

- Welche Probleme könnten in einer Help-line bzw. elektronisch mit den Hausärzten abgehandelt werden, ohne die ganze Bürokratie eines Grossspitals in Anspruch nehmen zu müssen?
Organisation ohne weiteres möglich. Es ist nicht die Begrenzung der Arbeitszeit, die verhindert, dass eine interessante Operation, die abends noch reinkommt, gemacht werden kann, sondern das Eingebunden-Sein in die Dienstleistung am nächsten Tag. Wenn es wirklich um die Weiterbildung ginge, könnte der Assistenzarzt operieren und dafür am nächsten Tag später kommen oder frei nehmen. Dazu sind die Stellenpläne aber zu knapp bemessen, weil der Weiterbildungsaspekt dabei vernachlässigt wird.

\section{Die Kontinuität ist gefährdet,}

\section{dadurch passieren mehr Fehler}

Patienten haben das Recht auf einen Arzt oder eine Ärztin, der bzw. die gut informiert, bei wachem Verstand und nicht übermüdet ist. Kein Patient erwartet während 24 Stunden und während seines ganzen Aufenthaltes die gleichen Betreuungspersonen, auch nicht den gleichen Arzt. Wichtig sind gute Übergaben und ein funktionierendes Klinikinformationssystem.

\section{Ein guter Arzt zählt seine Arbeitsstunden nicht, er ist bereit, für seine Patienten rund um die Uhr im Einsatz zu sein} Auch Ärztinnen und Ärzte haben begrenzte Kräfte und sie haben ein Recht auf ausreichende Erholung, auf ein intaktes Sozialleben und auf Freizeit. Das erhöht ihre Leistungsfähigkeit und ihre Sozialkompetenz. In anderen Berufen mit hoher Verantwortung sind strikte Begrenzungen der Arbeitszeit eine Selbstverständlichkeit. 